\title{
Default activity patterns at the neocortical microcircuit level
}

\author{
Artur Luczak ${ }^{1 *}$ and Jason N. MacLean ${ }^{2}$. \\ ${ }^{1}$ Department of Neuroscience, Canadian Centre for Behavioural Neuroscience, University of Lethbridge, Lethbridge, AB, Canada \\ 2 Department of Neurobiology, The University of Chicago, Chicago, IL, USA
}

\section{Edited by:}

Jeremy Seamans, University of

British Columbia, Canada

\section{Reviewed by:}

Kari L. Hoffman, York University, Canada

Tim Murphy, University of British Columbia, Canada

\section{${ }^{*}$ Correspondence:}

Artur Luczak, Department of

Neuroscience, Canadian Centre for Behavioural Neuroscience, University of Lethbridge, 4401 University Drive, Lethbridge, AB, Canada T1K 3M4. e-mail: luczak@uleth.ca;

Jason N. MacLean, Department of

Neurobiology, The University of

Chicago, 947 East 58th Street,

MC 0926, Chicago, IL 60637, USA.

e-mail: jmaclean@uchicago.edu
Even in absence of sensory stimuli cortical networks exhibit complex, self-organized activity patterns. While the function of those spontaneous patterns of activation remains poorly understood, recent studies both in vivo and in vitro have demonstrated that neocortical neurons activate in a surprisingly similar sequential order both spontaneously and following input into cortex. For example, neurons that tend to fire earlier within spontaneous bursts of activity also fire earlier than other neurons in response to sensory stimuli. These "default patterns" can last hundreds of milliseconds and are strongly conserved under a variety of conditions. In this paper, we will review recent evidence for these default patterns at the local cortical level. We speculate that cortical architecture imposes common constraints on spontaneous and evoked activity flow, which result in the similarity of the patterns.

Keywords: neocortex, microcircuit, spontaneous, imaging, tetrode, default mode

\section{INTRODUCTION}

Spontaneous activity is a principal mode of operation of the brain. It is defined as neuronal activity that is not directly tied to either sensory input or a behavioral task. Functionally, it has been suggested that cortical spontaneous activity underlies processes such as mental imagery (Kreiman et al., 2000; Kosslyn et al., 2001; Kraemer et al., 2005), cognition (Sadaghiani et al., 2010), and the consolidation of memories (Buzsaki, 1989; Hoffman and McNaughton, 2002; Born et al., 2006). In recent years, a variety of recording techniques across multiple in vivo and in vitro models have documented a resemblance of stimulus-evoked activity patterns to those that occur spontaneously. Considering the synaptic requirement for propagation of neuronal activity, it is likely that cortical connectivity imposes common constrains on the activity structure seen within a cortical circuit.

The initial description of a significant overlap between spontaneous and evoked activity patterns was provided by studies using voltage-sensitive dyes. Visualizing ongoing activity in cat visual cortex, Kenet et al. (2003) showed that spontaneously emerging patterns of activity corresponded closely to functional orientation maps. Similarly, patterns of activity emerging in response to sensory stimulation were also found to occur spontaneously in mouse sensorimotor cortex (Ferezou et al., 2006). Theoretical studies have shown a direct link between the connectivity in network models and the resultant dynamics (e.g., Honey et al., 2007; Galán, 2008; Roxin, 2011). Consistent with these theoretical studies, recent experiments using either ultrastructural analysis (Bock et al., 2011) or paired patch clamp recording (Ko et al., 2011) have demonstrated that visually evoked neuronal activity patterns reflected synaptic connectivity.

On a larger scale, Mohajerani et al. (2010) have shown that fine scale spontaneous activity patterns are mirrored between hemispheres and are the direct result of bilateral connectivity. Modification of this connectivity results in a dramatic reduction in the coherence of patterns between hemispheres. By combining anatomical tracing and fMRI, Vincent et al. (2007) provide evidence that both spontaneous and evoked patterns could be the byproduct of connectivity. Specifically, they found that "the pattern of saccade task-evoked activations resembles the distribution of spontaneous BOLD correlations in the oculomotor system" and showed that the correlation structure of spontaneous BOLD fluctuations relates to the underlying anatomical circuitry by using retrograde tracer injections.

The interdependence of spontaneous and evoked activity is further supported by compelling evidence of plastic remodeling of spontaneous activity by sensory experience. Using voltagesensitive dye imaging in rat visual cortex, Han et al. (2008) found that repetitive presentation of a visual stimulus modified ongoing spatiotemporal activity patterns such that these patterns more closely resembled the evoked responses. Thus, the overlap in activity patterns may be the product of intracortical plasticity mechanisms, suggesting that the similarity between spontaneous and evoked patterns is the product of dynamic remodeling of the underlying synaptic connectivity. In more global framework, spontaneous activity can be seen as an internal model of the learned sensory environment (Fiser et al., 2010). 
The most distinguishable patterns of spontaneous spiking activity are observed during slow wave oscillations (SWO), which can be observed during slow wave sleep (Metherate and Ashe, 1993; Steriade et al., 1993, 2001), quiet wakefulness (Petersen et al., 2003; Crochet and Petersen, 2006; Ferezou et al., 2006; Luczak et al., 2007, 2009), and under anesthesia (Steriade et al., 1993). SWO can also originate without pharmacological manipulation in vitro in slices of isolated cortex (Sanchez-Vives and McCormick, 2000; Cossart et al., 2003; Shu et al., 2003), showing a strong generalization of this rhythmic neuronal behavior to the cortex. During SWO, bursts of population activity called UP states last for $100 \mathrm{~ms}$ to several seconds and are interspersed with periods of neuronal silence (DOWN states; Metherate et al., 1992; Steriade et al., 1993; see example in Figure 1 middle column and in Figure 2A). UP states, whether spontaneous or evoked by external stimuli, occur simultaneously in nearby neurons (Lampl et al., 1999; Luczak et al., 2007, 2009) and exhibit complex spatiotemporal patterns of neuronal activity (Cossart et al., 2003; MacLean et al., 2005; Watson et al., 2008). Multiple studies have shown that those patterns occurring during spontaneous UP states are particularly similar to patterns produced by thalamic or sensory stimulation (Tsodyks et al., 1999; Kenet et al., 2003; Fiser et al., 2004; MacLean et al., 2005; Eggermont, 2006; Watson et al., 2008; Luczak et al., 2009). These data suggest that spontaneous patterns resemble stimulus evoked patterns because they propagate through the same microcircuits, and the architecture of synaptic weights and connections imposes significant 'hardware' constraints on activity patterns.

In this mini review, we will focus on the similarity of spontaneous and evoked activity patterns at the local circuit level. Although this similarity has also been observed on much larger spatial scales (Vincent et al., 2007; Mohajerani et al., 2010), we will focus on microcircuits that can be densely recorded from with single cell resolution. We term local patterns of neuronal activations that frequently repeat spontaneously or in response to stimuli as "default patterns" due to the preservation of their structure regardless of the source of initiation. Consistent with the theory that local cortical architecture plays a major role in generating these default patterns, we also introduce the term "default microcircuits." Thus, default microcircuits give rise to default patterns, reflecting the concept that specific connectivity in a local network constrains and shapes the spontaneous and evoked activity. We speculate that default microcircuits are a network of strongly interconnected neurons embedded in network of weaker connections (Song et al., 2005; Perin et al., 2011), likely shaped by plasticity mechanisms (Han et al., 2008). These strong connections cause spontaneous or evoked signals to be more likely to travel along these stronger connections, which, in turn, results in similar activity patterns.

\section{DEFAULT PATTERNS: IN VITRO}

Far from being random, spontaneous circuit activity is precisely patterned in terms of the timing of a specific neuron within a sequence of neuronal activity (Cossart et al., 2003; Kenet et al., 2003; MacLean et al., 2005; Watson et al., 2008; Luczak et al., 2007, 2009). Further, the same circuits that are spontaneously active in sensory cortices can also be activated by thalamic input in vitro (Castro-Alamancos, 2009; MacLean et al., 2005; Watson et al., 2008). Here we define a circuit as a group of neurons that are likely synaptically interconnected and functionally related (Bock et al., 2011; Ko et al., 2011). Thus, any discrete population of neurons that are co-active (i.e., a circuit) will be repeatedly co-active to the exclusion of the majority of the surrounding neurons. This suggests that this activity is the byproduct of discrete/specific synaptic connectivity inherent to cortex (Sanchez-Vives and McCormick,
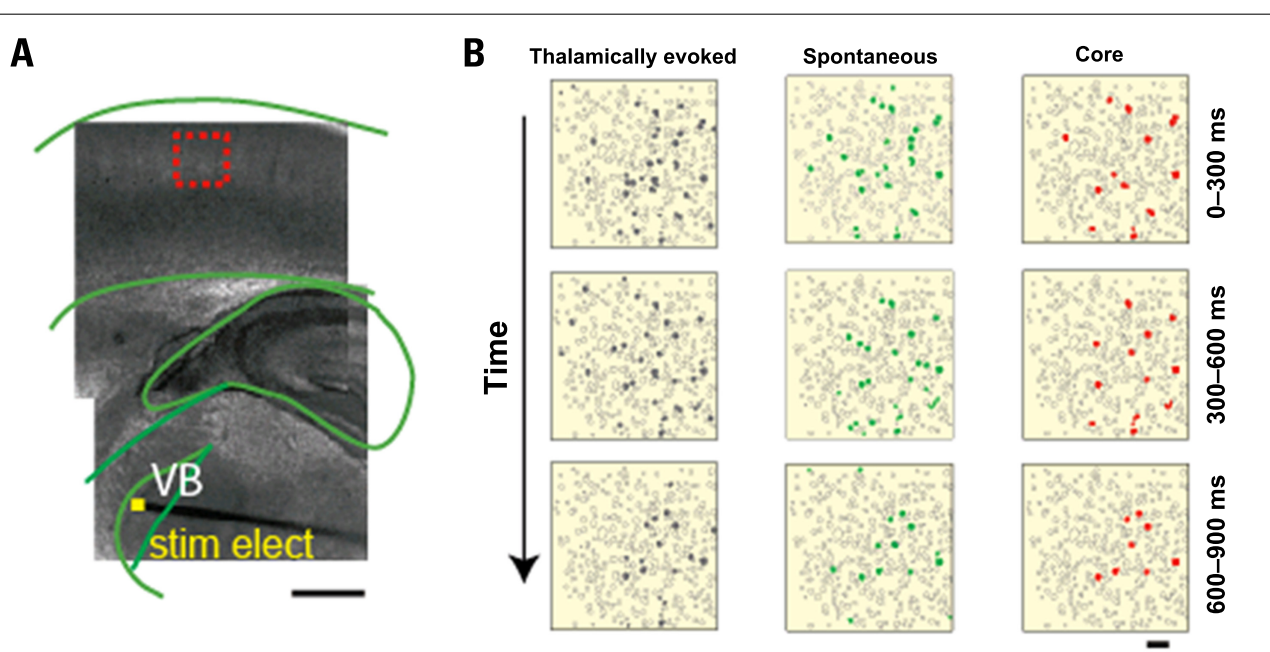

FIGURE 1 | Spatiotemporal precision of network activation: cells are activated in similar order spontaneously and following thalamic stimulation. (A) Light micrograph, with an overlaid cartoon, of a somatosensory (S1) thalamocortical slice preparation with intact thalamic input nucleus (ventral basal nucleus, VB), thalamocortical axons, and the somatosensory cortex. A stimulating electrode is placed in $\mathrm{VB}$, as indicated by yellow square. The superimposed dashed-red box indicates the location, over layer 4, of the illustrated frame in (B). Scale bar, $1 \mathrm{~mm}$. (B) Individual frames $(300 \mathrm{~ms}$ ) from representative movies of a thalamically evoked network activation (triggered, gray, left) and a spontaneous network activation (spontaneous, green, middle) in the same slice. Each movie progresses from top to bottom as indicated by the arrow. Core frames indicate cells active in the same order across all movies $(n=11)$ from this slice, indicated in red. Scale bar $50 \mu \mathrm{m}$ (adapted from MacLean et al., 2005). 
A

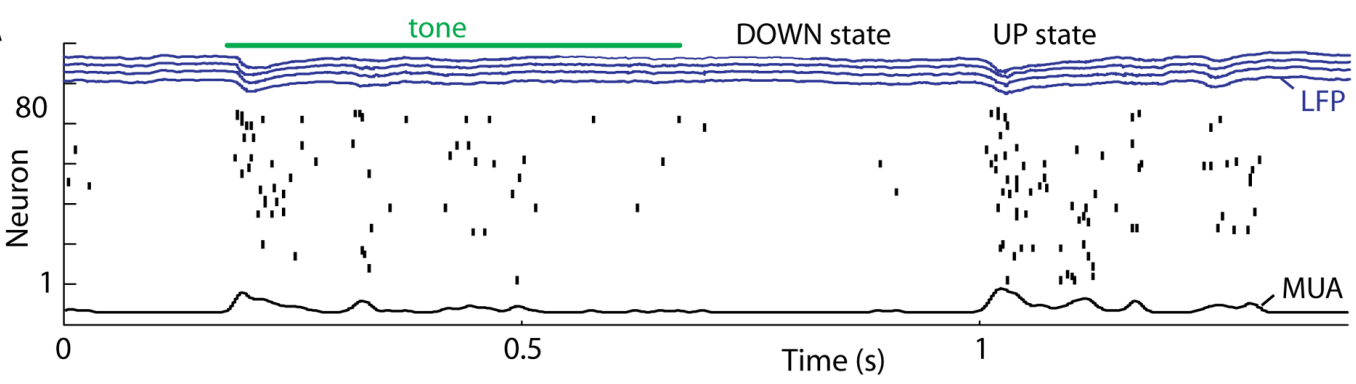

B

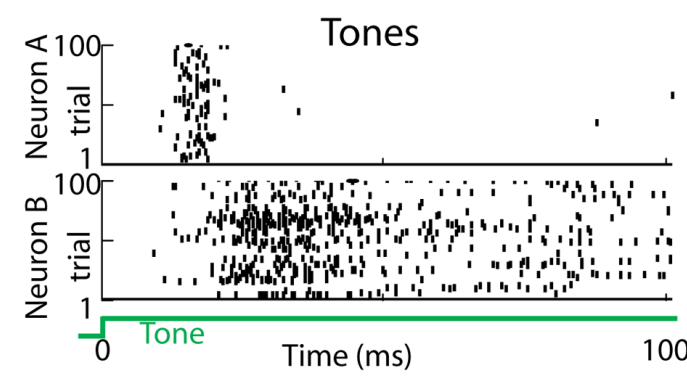

C

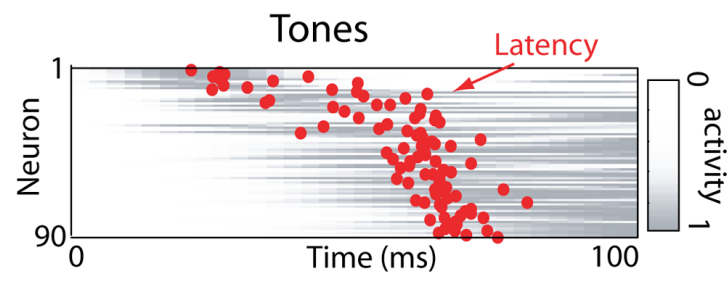

E
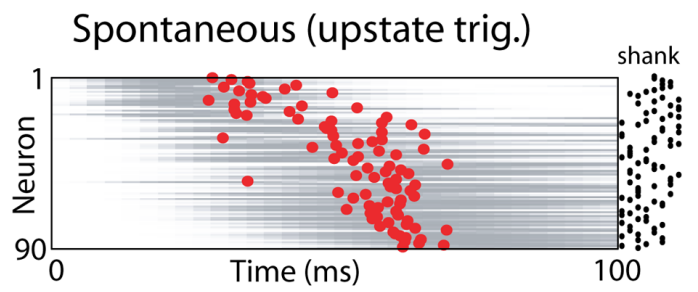

G

$\mathrm{F}$

Time (ms)

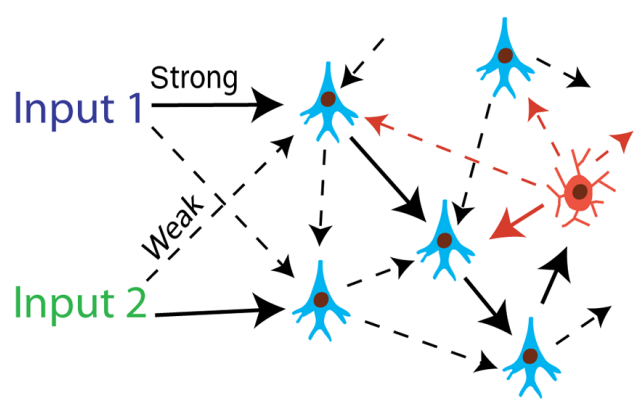

FIGURE 2 | Spontaneous UP states initiate sequential patterns homologous to evoked responses. (A) Representative raw data plot showing a tone response and spontaneous firing event. DOWN states of complete silence alternate with UP states of generalized activity. Neurons are ordered vertically by the mean latency over all stimuli, to illustrate sequential spread of activity. Blue traces show local field potentials (LFPS) from four separate recording shanks; at bottom is the multiunit firing rate (MUA). (B) Raster plots showing spike times for two representative neurons to repeated presentations of a pure tone stimulus. (C) Average activity of 90 simultaneously recorded neurons to tone stimuli. Gray bars show pseudocolor representations of each neuron's perievent time histogram normalized between 0 and 1; red dots denote each neuron's latency in the $100 \mathrm{~ms}$ after tone onset. (D) Response of the same two neurons as in (B) triggered by UP state onsets. Note the similar temporal pattern. (E) Average upstate-triggered activity of all neurons, sorted in the same order as in C (adapted from Luczak etal., 2009). (F) Cartoon illustration of default microcircuits - strongly connected neurons (solid arrows) embedded in pool

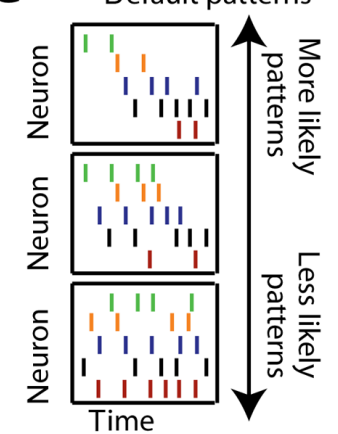

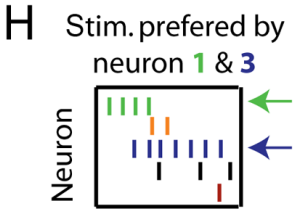

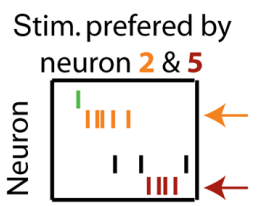

Time after stim. onset of weaker connections (dashed arrows). Due to constraints on connectivity, different inputs may result in similar activity propagation through the network. Most typical patterns produced in such default microcircuit are termed default patterns (G). (H) Cartoon illustration of stimulus-evoked patterns. The overall structure of evoked patterns is similar to the spontaneous default patterns shown in panel (G), but the firing rate and to smaller degree spike timing of neurons encodes information about stimulus identity. For example: during spontaneous activity neuron 1 (green) tends to fire before neuron 2 (brown). In response to a stimulus that is preferred by neuron 1 , neuron 1 fires at a higher rate $(4 \mathrm{~Hz})$ and with shorter delay $(5 \mathrm{~ms})$ after stimulus onset while neuron 2 fires at $2 \mathrm{~Hz} 25 \mathrm{~ms}$ after onset. For a different stimulus that is preferred by neuron 2, neuron 1 fires at a lower rate $(1 \mathrm{~Hz})$ and with longer delay $(15 \mathrm{~ms})$, while neuron 2 increases its firing rate by $3 \mathrm{~Hz}$ and shortens its delay by $5 \mathrm{~ms}$. This exemplifies how external stimulation can evoke a stimulus-specific firing rate and timing of neurons and still maintain the overall structure of the default pattern (i.e., neuron 1 fires before neuron 2). 
2000; Ko etal., 2011). Using mouse somatosensory thalamocortical slices and calcium imaging to observe action potential generation within individual neurons, it has been demonstrated that thalamic stimulation activates specific neocortical circuits, which repeatedly involve a particular population of neurons active in a particular sequence. These thalamically recruited circuits are statistically indistinguishable in the numbers, identities, and sequences of neurons firing during spontaneous activations of the same circuit (MacLean et al., 2005; Watson et al., 2008). This is especially the case when examining "core" circuit neurons, that is, neurons that participate in every activation of the specific circuit that they were part (MacLean et al., 2005; Yassin et al., 2010). By comparing all possible pairs of core neuron sequences, it was found that the percent of core neurons that were activated in exactly the same order both spontaneously and following thalamic input to be around $70 \%$. These repeating sequences were significantly different from bootstrap reshuffled data sets in which cell identity was maintained and only the time of activation during a circuit event was changed (Figure 1). Thus, cortical circuits that are activated by thalamic input significantly overlap with the activity that arises in these same circuits spontaneously. This result implies that intracortical connectivity plays a dominant role in determining the cortical response to sensory input.

\section{DEFAULT PATTERNS: IN VIVO SENSORY CORTICAL CIRCUITS}

To investigate if precise spatiotemporal sequences of activation also occur in vivo, Luczak et al. (2009) recorded simultaneously from 40-100 neurons in layer $\mathrm{V}$ of rat auditory cortex using silicon microelectrodes in both urethane anesthetized and awake rats. In response to tone stimuli as well as spontaneously, neurons showed sequential temporal firing patterns (Figure 2A). If the sequential structure of sensory responses is a reflection of a default dynamics of the circuit producing it, spontaneous patterns generated by the same circuit should show the same stereotyped sequential structure as sensory responses. In support of this prediction, individual neurons showed similar temporal relationships of their spiking activity to UP state onsets as they did to sensory stimuli, revealing a similar sequential structure at the population level (Figures 2B-E). The similarity of spontaneous and evoked patterns was also observed in somatosensory and visual areas (Jermakowicz et al., 2009; Luczak et al., 2009) and similar sequential patterns were also reported in prefrontal cortex (Peyrache et al., 2010). This suggests that default patterns are present in most cortical areas. Moreover, weak pair-wise correlations in neuronal circuits may cause major constraints not only on sequential structure of default patterns but also on firing rate correlations at the population level (Schneidman et al., 2006). Thus, default patterns not only demonstrate fine-scale temporal patterns, but also have similar firing rate correlations for both spontaneous and stimulus-evoked events (Luczak et al., 2009). These are important findings because it shows that population spike patterns in anesthetized and awake animals are much less diverse than previously assumed.

\section{DISCUSSION}

It is not surprising that the neuronal population patterns may show a certain level of similarity to each other, since more strongly connected neurons will be more likely to fire together across different conditions. Rather, the surprise is how highly conserved these activity patterns are under a variety of conditions. Considering that each cortical neuron receives input from potentially thousands of other neurons, any evoked or spontaneous activity pattern could have very different spatiotemporal dynamics from all other patterns. Contrary to this expectation, studies reviewed here show that neuronal responses are limited to a small subset of all possible activity patterns. We suggest that these "default patterns" are the functional manifestation of "default microcircuits" - local patterns of connectivity that impose similar spatiotemporal constraints on spontaneous and stimulus-evoked flow of activity, as illustrated in cartoon form in Figures 2F-H.

One profound question which comes to mind is: what would be the function of default patterns? We see it a little differently - that the system has to generate default patterns given the constraints imposed by synaptic connectivity. Thus, we feel it could be misguided to try to assign a specific function to this activity, rather default patterns reflect the circuit wiring diagram(s) in neocortex. Let's use an analogy: the arm is composed of set of bones and joints which put together set constraints on possible movements. Thus, although spontaneous arm movements, reaching for a cup or writing are quite different actions - patterns of muscles activity during those actions share many similarities, because it uses the same "hardware." Thus, default activity patterns are likely the manifestation of "hardware" constraints within the system. However, we believe that it is important to discuss the existence of default patterns because it can shed light on the structure of baseline activity, structure of stimulus-evoked patterns and thus will likely prove critical to our understanding of the informational coding scheme in cortex.

Although, results reviewed here indicate that the spatiotemporal population spike patterns are much less diverse than previously assumed, neuronal patterns are not carbon copies of one another. It is particularly important to keep in mind that although default microcircuits constrain neuronal activity dynamics, the number of possible patterns is still enormous, allowing for the unique representation of different stimuli (Luczak et al., 2009). For instance, for a preferred stimulus, a neuron will tend to respond with higher firing rate and with slightly shorter latency but the overall structure of default pattern will be preserved (Figure $\mathbf{2 H}$, see also Figure 1 in Luczak etal., 2009). It is also interesting to note that the highest precision of spike patterns is observed immediately after stimulus (Churchland etal., 2010) or UP state onset, after which timing precision progressively deteriorates (Luczak et al., 2007). Thus, those results are not fully consistent with concept of "synfire chains" which generally implies repeating patterns to have a millisecond-level precision for the entire duration of pattern (Abeles, 1991). It is conceivable that the reason for the highest precision of spiking observed immediately after onset could be that neurons firing earliest in the sequence would reflect an initial processing of information; and spiking activity at later times would reflect subsequent computations combined with feedback information from other areas. For example in several sensory systems, short-latency responses correlate with simple stimulus features, while later responses evolve to represent more complex 
features (Grastyan et al., 1978; Sugase et al., 1999; Brincat and Connor, 2006; Bartho et al., 2009). For this reason we feel that data reviewed here best fits with the theoretical construct based on cell assembly hypothesis. This idea introduced by Donald Hebb in the 1940s (Hebb, 1949) proposes that neurons are active collectively in groups produced by Hebbian plasticity. Further, Hebb postulates that different stimuli are represented by unique neuronal assemblies with completely different temporal patterns depending on task or stimulus. However, evidence for default patterns necessitates a partial revision of Hebb's theory. Specifically, conserved activity patterns imply that neuronal assemblies are like a variation on a one master theme rather than unique themes for each stimulus or object. For example, neurons in auditory cortex respond with similar temporal sequences to different tones (Figures 2B,C), although each tone evokes different variation of that pattern (Luczak et al., 2009).

The discovery of resting brain state in fMRI studies has required neuroscientists to rethink our understanding of "baseline activity" (Gusnard and Raichle, 2001). Similarly, the existence of default patterns should lead to more careful rethinking of baseline activity in electrophysiological experiments. For example, from the presented studies it is clear that assumption of spiking independence (e.g., Poisson process), or quiescence for that matter, is not an accurate description of neuronal population activity at rest. Spontaneous brain activity is full of structured patterns and ignoring this fact may lead to incorrect interpretation of experimental data especially when investigating temporal and firing rate relationships between neurons.

Despite the fact that many labs have observed and reported repeating patterns in neuronal activity, these results are not widely accepted. One of the reasons for this is the statistical difficulty in assessing the significance of reoccurring patterns. Specifically, the problem resides in defining a null hypothesis: what is the expected probability of a pattern arising by chance (Ikegaya et al., 2004; Mokeichev etal., 2007; Ikegaya et al., 2008; Roxin et al., 2008)? For example, should the analyzed spike trains be compared to a homogeneous or inhomogeneous Poisson process; if inhomogeneous, what then should be an appropriate modulation function? We would like to note that evidence showing consistent and repeating sequential neuronal activity in response to stimulation as well as to DOWN-UP state transition as reviewed here are not subject to these statistical difficulties.

Another potential source for discrepancy between results may be due to differences in brain state. For instance, during sleep, periods of SWO are interleaved with periods of REM sleep. Each

\section{REFERENCES}

Abeles, M. (1991). Corticonics: Neural Circuits of the Cerebral Cortex. New York: Cambridge University Press.

Bartho, P., Curto, C., Luczak, A., Marguet, S. L., and Harris, K. D. (2009). Population coding of tone stimuli in auditory cortex: dynamic rate vector analysis. Eur. J. Neurosci. 30, 1767-1778.

Bock, D. D., Lee, W. C. A., Kerlin, A. M., Anderman, M. L., Hood, G., Wetzel, A. W., Yurgenson, S., Soucy,

of those states has quite different dynamics. Moreover, the awake state is different from sleep and has its own range of states ranging from an animal at rest to an animal which is fully engaged while performing a task. Probably during "quiescent" states (i.e., SWO in thalamocortical slices, SWO in animals under anesthesia, "quiet" wakefulness; Petersen et al., 2003) it is easier to detect default activity patterns as opposed to the activated/attending state observed in animals engaged in task. We speculate that in the more active brain, i.e., attentive wakefulness, spatial and temporal overlap of patterns propagating through the same default microcircuits may obscure and complicate detection of any structured activity. By analogy, in slowly moving carousel it easy to see each of the seats, but when the carousel is spinning fast we can no longer distinguish single seats from the blur of motion. Thus, the apparent absence of patterns reported in some studies (e.g., Ecker et al., 2010) could be the result of a more activated brain state. In studies that densely sample from neuronal populations, as afforded by 2-photon imaging methods, significant correlation between nearby and task related neurons has been observed even during the activated state (e.g., Komiyama et al., 2010). This observation was made possible by imaging approaches that allow single cell resolution. Without fine spatial resolution, task-engaged circuits can be intermingled and overlapping, making it difficult to detect meaningful correlations and preserved spatial-temporal structure of local circuits. The other possible source of discrepancies in detecting repeated patterns may be found in the size of the time bin over which correlation is calculated, as it can lead to negative results if the time bin is too large or small. Regardless, we suggest that it is of utmost importance that the role and activity of neurons that comprise a default microcircuit be characterized across different brain states ranging from deep anesthesia to awake task-engaged animal.

In summary, we describe current evidence for existence of default patterns. We suggest that default microcircuits (strongly connected neurons embedded in pool of weaker connections) could cause similar propagation of activity through the network, despite differences in spontaneous and stimulus-evoked inputs (Figure 2F). As the result, certain types of activity patterns (i.e., default patterns) are more prominent and more frequent (Figure 2G).

\section{ACKNOWLEDGMENTS}

This work was supported by AHFMR (Artur Luczak) and NSERC (Artur Luczak). We also thank A. Renart, and M. Runfeldt for comments.

'noisy' brain states. Neuroscience 31, 551-570.

Castro-Alamancos, M. A. (2009). Cortical up and activated states: implications for sensory information processing. Neuroscientist 15, 625-634.

Churchland, M. M., Yu, B. M., Cunningham, J. P., Sugrue, L. P., Cohen, M. R., Corrado, G. S., Newsome, W. T., Clark, A. M., Hosseini, P., Scott, B. B., Bradley, D. C., Smith, M. A., Kohn, A., Movshon, J. A.,
Armstrong, K. M., Moore, T., Chang, S. W., Snyder, L. H., Lisberger, S. G., Priebe, N. J., Finn, I. M., Ferster, D., Ryu, S. I., Santhanam, G., Sahani, M., and Shenoy, K. V. (2010). Stimulus onset quenches neural variability: a widespread cortical phenomenon. Nat. Neurosci. 13, 369-378.

Cossart, R., Aronov, D., and Yuste, R. (2003). Attractor dynamics of network UP states in the neocortex. Nature 423, 283-288. 
Crochet, S., and Petersen, C. C. (2006). Correlating whisker behavior with membrane potential in barrel cortex of awake mice. Nat. Neurosci. 9, 608-610.

Ecker, A. S., Berens, P., Keliris, G. A., Bethge, M., Logothetis, N. K., and Tolias, A. S. (2010). Decorrelated neuronal firing in cortical microcircuits. Science 327, 584-587.

Eggermont, J. J. (2006). Properties of correlated neural activity clusters in cat auditory cortex resemble those of neural assemblies. J. Neurophysiol. 96, 746-764.

Ferezou, I., Bolea, S., and Petersen, C. C. (2006). Visualizing the cortical representation of whisker touch: voltage-sensitive dye imaging in freely moving mice. Neuron 50, 617-629.

Fiser, J., Chiu, C., and Weliky, M. (2004). Small modulation of ongoing cortical dynamics by sensory input during natural vision. Nature 431, 573-578.

Fiser, J., Berkes, P., Orban, G., and Lengyel, M. (2010). Statistically optimal perception and learning: from behavior to neural representations. Trends Cogn. Sci. 14, 119-130.

Galán, R. F. (2008). On how network architecture determines the dominant patterns of spontaneous neural activity. PLOS ONE 3, e2148. doi: 10.1371/journal.pone. 0002148

Grastyan, E., John, E. R., and Bartlett, F. (1978). Evoked response correlate of symbol and significate. Science 201, 169-171.

Gusnard, D. A., and Raichle, M. E. (2001). Searching for a baseline: functional imaging and the resting human brain. Nat. Rev. Neurosci. 2, 685-694.

Han, F., Caporale, N., and Dan, Y. (2008). Reverberation of recent visual experience in spontaneous cortical waves. Neuron 60, 321-327.

Hebb, D. O. (1949). The Organization of Behavior. New York: Wiley.

Hoffman, K. L., and McNaughton, B. L. (2002). Coordinated reactivation of distributed memory traces in primate neocortex. Science 297, 2070-2073.

Honey, C. J., Kotter, R., Breakspear, M., and Sporns, O. (2007). Network structure of cerebral cortex shapes functional connectivity on multiple time scales. Proc. Natl. Acad. Sci. U.S.A. 104, 10240-10245.

Ikegaya, Y., Aaron, G., Cossart, R., Aronov, D., Lampl, I., Ferster, D., and Yuste, R. (2004). Synfire chains and cortical songs: temporal modules of cortical activity. Science 304, 559-564.

Ikegaya, Y., Matsumoto, W., Chiou, H. Y., Yuste, R., and Aaron, G.
(2008). Statistical significance of precisely repeated intracellular synaptic patterns. PLoS ONE 3, e3983. doi: 10.1371/journal.pone.0003983

Jermakowicz, W. J., Chen, X., Khaytin, I., Bonds, A. B., and Casagrande, V. A. (2009). Relationship between spontaneous and evoked spike-time correlations in primate visual cortex. J. Neurophysiol. 101, 2279-2289.

Kenet, T., Bibitchkov, D., Tsodyks, M., Grinvald, A., and Arieli, A. (2003). Spontaneously emerging cortical representations of visual attributes. Nature 425, 954-956.

Ko, H., Hofer, S. B., Pichler, B., Buchanan, K. A., Sjöström, P. J., and Mrsic-Flogel, T. D. (2011). Functional specificity of local synaptic connections in neocortical networks. Nature 473, 87-91.

Komiyama, T., Sato, T. R., O’Connor, D. H., Zhang, Y. X., Huber, D., Hooks, B. M., Gabitto, M., and Svoboda, K. (2010). Learning-related fine-scale specificity imaged in motor cortex circuits of behaving mice. Nature 464, 1182-1186.

Kosslyn, S. M., Ganis, G., and Thompson, W. L. (2001). Neural foundations of imagery. Nat. Rev. Neurosci. 2, 635-642.

Kraemer, D. J., Macrae, C. N., Green, A. E., and Kelley, W. M. (2005). Musical imagery: sound of silence activates auditory cortex. Nature 434, 158.

Kreiman, G., Koch, C., and Fried, I. (2000). Imagery neurons in the human brain. Nature 408, 357-361.

Lampl, I., Reichova, I., and Ferster, D. (1999). Synchronous membrane potential fluctuations in neurons of the cat visual cortex. Neuron 22, 361-374.

Luczak,A., Barthó, P., and Harris, K. D. (2009). Spontaneous events outline the realm of possible sensory responses in neocortical populations. Neuron 62, 413-425.

Luczak, A., Barthó, P., Marguet, S. L., Buzsaki, G., and Harris, K. D. (2007). Sequential structure of neocortical spontaneous activity in vivo. Proc. Natl. Acad. Sci. U.S.A. 104, 347-352.

MacLean, J. N., Watson, B. O., Aaron, G. B., and Yuste, R. (2005). Internal dynamics determine the cortical response to thalamic stimulation. Neuron 48, 811-823.

Metherate, R., and Ashe, J. H. (1993). Ionic flux contributions to neocortical slow waves and nucleus basalis-mediated activation: wholecell recordings in vivo. J. Neurosci. 13, 5312-5323.

Metherate, R., Cox, C. L., and Ashe, J. H. (1992). Cellular bases of neocortical activation: modulation of neural oscillations by the nucleus basalis and endogenous acetylcholine. J. Neurosci. 12, 4701-4711.

Mohajerani, M. H., McVea, D. A., Matthew Fingas, M., and Murphy, T. H. (2010). Mirrored bilateral slowwave cortical activity within local circuits revealed by fast bihemispheric voltage-sensitive dye imaging in anesthetized and awake mice. J. Neurosci. 30, 3745-3751.

Mokeichev, A., Okun, M., Barak, O., Katz, Y., Ben-Shahar, O., and Lampl, I. (2007). Stochastic emergence of repeating cortical motifs in spontaneous membrane potential fluctuations in vivo. Neuron 53, 413-425.

Petersen, C. C., Hahn, T. T., Mehta, M., Grinvald, A., and Sakmann, B. (2003). Interaction of sensory responses with spontaneous depolarization in layer $2 / 3$ barrel cortex. Proc. Natl. Acad. Sci. U.S.A. 100, 13638-13643.

Perin, R., Berger, T. K., and Markram, H. (2011). A synaptic organizing principle for cortical neuronal groups. Proc. Natl. Acad. Sci. U.S.A. 108 5419-5424.

Peyrache, A., Benchenane, K., Khamassi, M., Wiener, S. I., and Battaglia, F. P. (2010). Sequential reinstatement of neocortical activity during slow oscillations depends on cells' global activity. Front. Syst. Neurosci. 3:18. doi: 10.3389/neuro.06. 018.2009

Roxin, A. (2011). The Role of degree distribution in shaping the dynamics in networks of sparsely connected spiking neurons. Front. Comput. Neurosci. 5:8. doi: 10.3389/fncom. 2011.00008

Roxin, A., Hakim, V., and Brunel, N. (2008). The statistics of repeating patterns of cortical activity can be reproduced by a model network of stochastic binary neurons. J. Neurosci. 28, 10734-10745.

Sadaghiani, S., Hesselmann, G., Friston, K. J., and Kleinschmidt, A. (2010). The relation of ongoing brain activity, evoked neural responses, and cognition. Front. Syst. Neurosci. 4:20. doi: 10.3389/fnsys. 2010.00020

Sanchez-Vives, M. V., and McCormick, D. A. (2000). Cellular and network mechanisms of rhythmic recurrent activity in neocortex. Nat. Neurosci. 3, 1027-1034.

Schneidman, E., Berry, M. J., Segev, R., and Bialek, W. (2006). Weak pairwise correlations imply strongly correlated network states in a neural population. Nature 440, 1007-1012.

Shu, Y., Hasenstaub, A., and McCormick, D. A. (2003). Turning on and off recurrent balanced cortical activity. Nature 423, 288-293.

Song, S., Sjöström, P. J., Reigl, M., Nelson, S., and Chklovskii, D. B. (2005). Highly nonrandom features of synaptic connectivity in local cortical circuits. PLoS Biol. 3, e68. doi: 10.1371/journal.pbio.0030068

Steriade, M., McCormick, D. A., and Sejnowski, T. J. (1993). Thalamocortical oscillations in the sleeping and aroused brain. Science 262, 679-685. Steriade, M., Timofeev, I., and Grenier, F. (2001). Natural waking and sleep states: a view from inside neocortical neurons. J. Neurophysiol. 85 , 1969-1985.

Sugase, Y., Yamane, S., Ueno, S., and Kawano, K. (1999). Global and fine information coded by single neurons in the temporal visual cortex. Nature 400, 869-873.

Tsodyks, M., Kenet, T., Grinvald, A., and Arieli, A. (1999). Linking spontaneous activity of single cortical neurons and the underlying functional architecture. Science 286, 1943-1946.

Vincent, J. L., Patel, G. H., Fox, M. D., Snyder, A. Z., Baker, J. T., Van Essen, D. C., Zempel, J. M., Snyder, L. H., Corbetta, M., and Raichle, M. E. (2007). Intrinsic functional architecture in the anaesthetized monkey brain. Nature 447, 83-86.

Watson, B. O., MacLean, J. N., and Yuste, R. (2008). UP states protect ongoing cortical activity from thalamic inputs. PLoS ONE 3, e3971. doi: 10.1371/journal.pone.0003971

Yassin, L., Benedetti, B. L., Jouhanneau, J. S., Wen, J. A., Poulet, J. F., and Barth, A. L. (2010). An embedded subnetwork of highly active neurons in the neocortex. Neuron 68, 1043-1050.

Conflict of Interest Statement: The authors declare that the research was conducted in the absence of any commercial or financial relationships that could be construed as a potential conflict of interest.

Received: 12 December 2011; accepted: 24 May 2012; published online: 12 June 2012.

Citation: Luczak A and MacLean JN (2012) Default activity patterns at the neocortical microcircuit level. Front. Integr. Neurosci. 6:30. doi: 10.3389/fnint. 2012.00030

Copyright (c) 2012 Luczak and MacLean. This is an open-access article distributed under the terms of the Creative Commons Attribution Non Commercial License, which permits non-commercial use, distribution, and reproduction in other forums, provided the original authors and source are credited. 